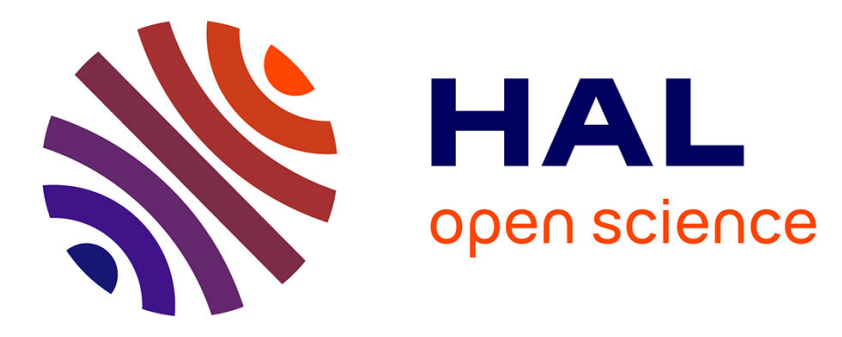

\title{
Spectroscopy of Covalently Functionalized Graphene
}

\author{
Sandip Niyogi, Elena Bekyarova, Mikhail E. Itkis, Hang Zhang, Kristin \\ Shepperd, J. Hicks, Mike Sprinkle, Claire Berger, Chun Ning Lau, Walt A. de \\ Heer, et al.
}

\section{- To cite this version:}

Sandip Niyogi, Elena Bekyarova, Mikhail E. Itkis, Hang Zhang, Kristin Shepperd, et al.. Spectroscopy of Covalently Functionalized Graphene. Nano Letters, 2010, 10, pp.4061-4066. 10.1021/nl1021128 . hal-01002908

\section{HAL Id: hal-01002908 https://hal.science/hal-01002908}

Submitted on 7 Jun 2014

HAL is a multi-disciplinary open access archive for the deposit and dissemination of scientific research documents, whether they are published or not. The documents may come from teaching and research institutions in France or abroad, or from public or private research centers.
L'archive ouverte pluridisciplinaire HAL, est destinée au dépôt et à la diffusion de documents scientifiques de niveau recherche, publiés ou non, émanant des établissements d'enseignement et de recherche français ou étrangers, des laboratoires publics ou privés. 


\title{
Spectroscopy of Covalently Functionalized Graphene
}

Sandip Niyogi,,,$\ddagger$ Elena Bekyarova,,,+ Mikhail E. Itkis,,$\uparrow$, Hang Zhang, Kristin Shepperd,,$\perp$ Jeremy Hicks,, Michael Sprinkle,, Claire Berger $, \perp, \sharp$ Chun Ning Lau,,,$\downarrow$ Walt A. deHeer,,$\perp$ Edward H. Conrad, $\perp$ and Robert C. Haddon,,$+\neq, \&$

${ }_{\dagger}$ Center for Nanoscale Science and Engineering, ${ }_{-D e p a r t m e n t}$ of Chemistry, §Department of Chemical and Environmental Engineering, and Department of Physics and Astronomy, University of California, Riverside, California 92521

${ }_{\perp}$ School of Physics, Georgia Institute of Technology, Atlanta, Georgia 30332,

\#CNRS/Institut Néel, BP166, 38042 Grenoble, France

\begin{abstract}
The atomically flat surface of graphene provides an opportunity to apply carbon-carbon bond-forming chemical reactions to engineer the electronic properties of graphene circuitry. In particular, covalent functionalization of the surface or the edge of graphene ribbons provides a novel way to introduce patterning that can modulate the energy band gap, affect electron scattering, and direct current flow by producing dielectric regions in a graphene wafer. We discuss the use of Raman spectroscopy and scanning tunneling microscopy to characterize the surface functionalization periodicities and densities that have been produced by the chemical derivatization of epitaxial graphene together with the concomitant changes in the electronic and magnetic properties of the graphene surface layer.
\end{abstract}


The construction of electronic circuitry based on the conjugated graphene lattice depends on the development of methods for nanopatterning of graphene wafers. ${ }^{1}$ Most obviously this would allow the fabrication of individual devices and wiring within the graphene wafer in the same way that a silicon wafer is patterned in a semiconductor foundry. In the case of graphene, however, the patterning is necessary to develop a band gap; in a perfect graphene sheet the valence and conduction bands touch at the Fermi level, and thus such structures cannot be efficiently switched to a low conductance state and the application of a gate voltage produces small changes in the device output. ${ }^{2,3}$

Graphene band gap engineering efforts are mainly focused on the use of lithographic methods to dice the graphene lattice into nanoribbons which physically confine the carriers. - $^{-6}$ However, the lithography process involves high-energy electron beams that has been shown to alter the intrinsic chemical structure of graphene. ${ }^{7}$ The demonstration that physical methods can open a band gap in graphene has also led to efforts in synthesizing graphene nanoribbons. ${ }^{8-10}$

We have focused on the application of chemistry to engineer a band gap into graphene; ${ }^{11,12}$ covalent carbon-carbon bond formation reactions can be used to change the hybridization of the graphitic atoms from $\mathrm{sp} 2$ to $\mathrm{sp} 3$ to modify the conjugation length of the delocalized carbon lattice. Such chemistry has been shown to be effective in modifying the electronic structure of single-walled carbon nanotubes (SWNTs), and these side-wall reactions serve to introduce a band gap in metallic SWNTs. ${ }^{13,14}$

Here we show that the covalent functionalization discussed above produces characteristic and clearly discernible modifications to the Raman spectra of graphene. The inplane vibrations of the conjugated $\boldsymbol{\pi}$-bonds have characteristic Raman spectrum when excited with visible light, and we show that the frequency of the Raman D-band as a result of the chemical functionalization on the graphene basal plane is distinct from that due to localized, structural defects in $\mathrm{sp}^{2}$ conjugated carbon. We show that this characteristic is found in exfoliated as well as epitaxial graphene and identify the distinguishing features between the different forms of graphitic carbon. The observations are interpreted as a demonstration of the application of covalent bond forming chemistry to affect the conjugation length and periodicity in graphene that results in the $\sim 0.4 \mathrm{eV}$ band gap measured using angleresolved photoelectron spectroscopy (ARPES).15,16

We begin by discussing the functionalization of exfoliated graphene because it gives rise to the simplest spectra; we found that the same functionalization scheme that we employed with epitaxial graphene wafers 11 is also effective in derivatizing exfoliated graphene on silicon substrates. The reaction occurs by spontaneous electron transfer from graphene to p-nitrobenzenediazonium tetrafluoroborate, which ultimately leads to the formation of a C-C bond between graphene and the nitrophenyl group; 
the hybridization of the graphene carbon atom is changed to $\mathrm{sp}^{3}$ in the process (Figure 1a). ${ }^{11}$

The Raman spectrum of single layer graphene (1-LG) has two characteristic peaks, the G-peak, which is due to the E2g vibrational mode of sp2 bonded carbon and is observed at $1580 \mathrm{~cm}^{-1}$, and the 2D peak (sometimes referred to as $\mathrm{G}^{\prime}$ ) at $2670 \mathrm{~cm}^{-1}$, which is a second order vibration caused by the scattering of phonons at the zone boundary; ${ }^{17,18}$ the intensity of the 2D peak is about three times the intensity of the G band (Figure 1b). After the chemical reaction, the D-band at $1336 \mathrm{~cm}^{-1}$ becomes the most prominent feature of the Raman spectrum (Figure 1b) and the characteristic 2D band intensity is significantly reduced. The D-band is due to the A1g mode breathing vibrations of six-membered sp2 carbon rings, and becomes Raman active after neighboring $\mathrm{sp} 2$ carbons are converted to $\mathrm{sp}^{3}$ hybridization in graphitic materials. Polycyclic aromatic hydro-carbons show Raman A1g mode peaks at $\sim 1300 \mathrm{~cm}^{-1}$ and Eg modes at $\sim 1500 \mathrm{~cm}^{-1} ; 19$ however, these structures have not been experimentally relevant as graphene derivatives before the current work. The observation of the Dband is accompanied by the $\mathrm{D}^{*}$ band at $1615 \mathrm{~cm}^{-1}$ and the $\mathrm{D}+\mathrm{D}^{*}$ band around 2930 $\mathrm{cm}^{-1}$. In the product, the 2D band is a single peak at $2670 \mathrm{~cm}^{-1}$ but the fwhm is $41 \mathrm{~cm}^{-}$ ${ }^{1}$ compared to $26 \mathrm{~cm}^{-1}$ in graphene. In graphene and graphitic materials, the widening of the 2D peak is associated with a frequency blue shift of $20-30 \mathrm{~cm}^{-1}$ (graphene to graphite), and it is related to interlayer electronic interactions, splitting into two peaks in graphite and four peaks in bilayer graphene. ${ }^{17}$ We note that under identical conditions HOPG samples did not give the same results and it appears that the product structure responsible for the Raman features described above are specific to graphene; in fact, even bilayer graphene (2-LG) samples behave differently. ${ }^{20,21}$

In the bilayer graphene sample (Figure 1c), the 2D band can be fitted with four Lorentzians (Figure 1c inset). The D, D*, D + D* bands in the product are broad and weak although they are observed at the same frequency as in the 1-LG samples (Figure 1d). The 2D band intensity is apparently reduced in the product (Figure $1 \mathrm{~b}, \mathrm{c})$; a similar decrease in the 2D band intensity was previously ascribed to $\mathrm{p}$-type doping of graphene which changes the resonance condition characteristic of the transition.22 The intensity of the band at $1510 \mathrm{~cm}^{-1}$ (Figure 1d) was found to increase with the D-band intensity and therefore is associated with graphene inplane vibrations but the bands at 1339 and $1442 \mathrm{~cm}^{-1}$ may originate from vibrations of the nitrophenyl ring. ${ }^{21-23}$

Epitaxial graphene grown either on the Si-face or on the C-face of hexagonal $\mathrm{SiC}$ may have domains of misoriented (rotationally disordered) and Bernal-stacked graphene. ${ }^{24-26}$ As shown in Figure 2a inset, domains can be visualized as shades of gray in an optical microscope but the length scales over which the Raman spectrum varies is much smaller than the optical images and hence it is difficult to visually predict the Raman spectrum of an epitaxial graphene sample in the same manner as is possible for exfoliated samples. Typically, the brighter regions in the image show 
spectra similar to Figure $2 \mathrm{~b}$ and the darker regions show spectra similar to Figure 2c. A Raman spectrum with $\mathrm{G}$ band intensity greater than the 2D band intensity is the characteristic of Bernal stacking. In Figure $2 \mathrm{~b}$ (inset), the $2 \mathrm{D}$ band has been fitted to four Lorentzians $\left(2680,2705,2724\right.$, and $2746 \mathrm{~cm}^{-1}$ with fwhm of 50, 21, 35, and 22 $\mathrm{cm}^{-1}$, respectively), resembling a Bernal-stacked 2-LG electronic structure, similar to that in Figure 1c. The G band at $1586 \mathrm{~cm}-1$, however, has a fwhm of $15 \mathrm{~cm}^{-1}$, which is significantly narrower than that of graphite. Figure $2 \mathrm{c}$ shows the other characteristic Raman spectrum observed in our epitaxial graphene samples, which resembles the spectrum of graphene in Figure 1b, except, the G band observed at $1586 \mathrm{~cm}^{-1}$ has a fwhm of $14 \mathrm{~cm}^{-1}$ and the 2D band at $2701 \mathrm{~cm}^{-1}$ has a fwhm of $18 \mathrm{~cm}^{-1}$.

The two regions represented in Figure 2b,c can be considered as the extremes in the three-dimensional spatial distribution (on the surface as well as in the $\mathbf{c}$-axis direction) of G and 2D band intensities over the $3.5 \mathrm{~mm}$ x $4.5 \mathrm{~mm}$ epitaxial graphene wafers. This distinguishes the Raman characteristics of epitaxial graphene from graphene and graphite. When the graphene layers are misoriented, it has been shown that the band structure can be treated as that of a single layer graphene. ${ }^{25,27,28}$ As in the exfoliated samples, the D-band is the most prominent peak in nitrophenyl (NP) functionalized epitaxial graphene but it is observed at an even lower energy between $1320-1330 \mathrm{~cm}^{-1}$ and the $\mathrm{D}^{*}\left(1601 \mathrm{~cm}^{-1}\right)$ and $\mathrm{D}+\mathrm{D}^{*}$ bands appear simultaneously.

Transport measurements and theoretical calculations imply the presence of an energy band gap in sp3 functionalized graphene, ${ }^{11,12,29}$ and thus we investigated the effect of nitrophenyl functionalization on epitaxial graphene using angle-resolved photoemission spectroscopy (ARPES); Figure 3a shows the band structure at the graphene K-point. Although the spectra are diffuse, two clear bands are seen; the presence of multiple bands in Figure 3a are the result of two different rotated graphene sheets in the $40 \mu \mathrm{m}$ ARPES beam normally associated with thick C-face graphene films. ${ }^{11}$ After NP-functionalization the linear bands of graphene have been transformed into massive bands shifted approximately $0.36 \mathrm{eV}$ below the Fermi level. Constant energy cuts (Figure 3b) clearly show that a gap has opened in the film. Because ARPES only measures filled states below the Fermi level, it is not possible to precisely determine the gap energy without doping the sample. ${ }^{16}$ Nonetheless, the lower limit on the band gap is conservatively estimated as $0.36 \mathrm{eV}$; this gap is comparable to that observed for atomic hydrogen doped graphene on $\operatorname{Ir}(111){ }^{30}$

Intense and sharp D-bands as shown in Figures $1 \mathrm{~b}$ and 2a have previously been reported in graphene nanoribbons and in functionalized exfoliated graphene samples. ${ }^{7,21,31-33}$ The frequency of the D-band in these samples is around $1340 \mathrm{~cm}^{-1}$ with $\lambda_{\mathrm{EX}}=532 \mathrm{~nm}$ and around $1350 \mathrm{~cm}-1$ with $\lambda_{\mathrm{EX}}=514 \mathrm{~nm}$; the D-band frequency shifts with the excitation energy $\left(60 \mathrm{~cm}^{-1} / \mathrm{eV}\right) .{ }^{34}$ Disordered exfoliated samples, defects in pristine epitaxial graphene samples, and nanodiamond show a D-band between $1342-1347 \mathrm{~cm}^{-1}$ in our experiments. In physically defective graphitic materials, the ratio of the $D$ and $G$ band integrated intensities $\left(I_{D} / I_{G}\right)$ has been found to depend on the crystallite size (La), ${ }^{35,36}$ and this correlation has been extensively 
explored; in the original work the following relationship was reported: $\mathbf{I}_{\mathrm{D}} / \mathbf{I}_{\mathrm{G}}=\mathbf{C}_{\mathrm{L}}(\boldsymbol{\lambda})$ / La, where $\mathbf{C}_{\mathrm{L}}(\lambda)$ ) $4.4 \mathrm{~nm}$ for $\lambda=488 \mathrm{~nm} .{ }^{35} \mathrm{~A}$ more recent analysis found $\mathbf{I}_{\mathrm{D}} / \mathbf{I}_{\mathrm{G}}=$ $C_{\mathrm{L}}(\lambda) / \mathrm{LD}$, where $\mathbf{C}_{\mathrm{L}}(\lambda)=11 \mathrm{~nm}$ for $\lambda=514 \mathrm{~nm}$ and $\mathbf{L}_{\mathrm{D}}$ is the average distance between defects; $\mathbf{L}_{D}=\boldsymbol{\sigma}^{-1 / 2}$, where $\boldsymbol{\sigma}$ is the defect density. ${ }^{37}$ However, it is now recognized that the dependence of the intensities $\mathbf{I}_{\mathrm{D}} / \mathbf{I}_{\mathrm{G}}$ on the crystallite sizes $\mathbf{L a}$ is quite complex and the above equations only describe this relationship in the regime of large crystallite sizes, $\mathbf{L a}>5-20 \mathrm{~nm} .{ }^{36,37}$ At small values of La, the strength of the Dmode depends on the occurrence of conjugated six-membered rings in the graphitic structure, and in this regime the relationship $\mathbf{I}_{\mathrm{D}} / \mathbf{I}_{\mathrm{G}}=\mathbf{C}_{\mathrm{S}}(\lambda) \mathbf{L}_{\mathrm{D}}{ }^{2}$ was proposed in which $C_{S}(\lambda)=0.55 \mathrm{~nm}^{-2}$ for $\lambda=514 \mathrm{~nm}^{36,37}$

We previously estimated the saturation density of NPfunctionalized epitaxial graphene as $10^{15}$ molecules $/ \mathrm{cm}^{2} ; 11$ this is equivalent to a surface coverage of nitrophenyl groups in which $\sim 20 \%$ of the graphene carbon atoms are functionalized (converted to $\mathrm{sp} 3$ hybridization).12 Thus the density of $\mathrm{sp}^{3}$ carbon atoms is $\boldsymbol{\sigma} \sim$ $8 / \mathrm{nm}^{2}$, which gives $\mathbf{L}_{\mathrm{D}} \sim 0.35 \mathrm{~nm}$ and thus application of the equation $\mathbf{I}_{\mathrm{D}} / \mathbf{I}_{\mathrm{G}}=\mathbf{C}_{\mathrm{S}}(\lambda) \mathbf{L}_{\mathrm{D}}{ }^{2}$ (with $\mathbf{C}_{\mathrm{S}}(\lambda)=0.55 \mathrm{~nm}^{-2}$ ), ${ }^{36,37}$ gives $\mathbf{I}_{\mathrm{D}} / \mathbf{I}_{\mathrm{G}}=7 \times 10^{-2}$ in obvious disagreement with our results; $\mathbf{I}_{\mathrm{D}} / \mathbf{I}_{\mathrm{G}}=2.6$ for NP-functionalized exfoliated graphene as shown in Figures 1 and $4 ; \mathbf{I}_{\mathrm{D}} / \mathbf{I}_{\mathrm{G}}=2.6$ and 3.3 for NP-functionalized epitaxial graphene in Figures 2 and 4, respectively. We conclude that the chemically functionalized graphene that we discuss above is entirely distinct from the physically defective material that has been considered in the past. In fact, an analysis of ion induced damage to graphene, concludes that the defects themselves have a structurally disordered region of radius, $\mathbf{r}_{\mathrm{S}}=1 \mathrm{~nm}$, which in turn activates the $\mathrm{D}$-band in a surrounding region of radius, $\mathbf{r}_{\mathrm{A}}=3 \mathrm{~nm} ;{ }^{37}$ obviously the chemically induced modifications of the structure that we discuss occur on a much smaller length scale.

Thus we turned to an analysis of hydrogenated amorphous carbon (a-C:H);36 this material provides a more appropriate model for the chemistry that we have employed in that it contains sp3 hybridized C-H groups as a result of the saturation of sp2 carbon atoms by addition of hydrogen atoms which serves to open a band gap; this behavior is very similar to our process (above) and strongly related to the production of graphane.7,30,32 Furthermore as the sp3 content increases in (a-C:H), the band gap increases, 36 as noted in covalently functionalized graphene in both experimental7,12,30,32 and theoretical investigations. 29 We took selected data from the analysis of $(\mathrm{a}-\mathrm{C}: \mathrm{H}) 36$ and plotted the reciprocal of the fractional sp3 content [F(sp3)] against ID/IG (Supporting Information Figure S1), which allowed us to derive the following relationship: $\mathbf{I}_{\mathrm{D}} / \mathbf{I}_{\mathrm{G}}=0.65 / \mathrm{F}(\mathrm{sp} 3)$ (see Figure 4, inset), or $\mathbf{I}_{\mathrm{D}} / \mathbf{I}_{\mathrm{G}}=\mathbf{C}_{\mathrm{C}}(\boldsymbol{\lambda}) \mathbf{L}_{\mathrm{D}}{ }^{2}$ (with $\mathbf{C c}(\lambda)=25 \mathrm{~nm}^{-2}$ ), and thus we obtain ID $/ \mathbf{I} \mathrm{G} \sim 3.1$, which is in remarkably good agreement with our results, but entirely distinct from the values which are characteristic of structurally based physical defects in unfunctionalized samples.

Under thermodynamic control, theoretical calculations indicated a pairwise addition of functionalities to the graphene A and the B sublattices leading to the formation of formally spin-paired Kekule products with unit cell functionalization densities of 2:8 
(25\%; LD $0.32 \mathrm{~nm}),{ }^{29}$ and 2:18 (11\%; $\left.\mathrm{L}_{\mathrm{D}} \sim 0.49 \mathrm{~nm}\right),{ }^{38}$ which have been discussed in terms of the Clar representations of these limiting substitution patterns. ${ }^{12}$ The 2:8mode of addition is expected for small substituents but the steric bulk of the phenyl groups inhibits such a configuration that would require neighboring phenyl ring contacts below the van der Waals separation,,$^{29,38}$ and accounts for the fact that at saturation, the graphene coverage with nitrophenyl groups is below $25 \%\left[\mathrm{~F}\left(\mathrm{sp}^{3}\right)\right.$ $0.2, \mathrm{LD} \sim 0.35 \mathrm{~nm}] .{ }^{12}$ The calculated Raman frequencies and intensities of hydroxyl functionalized graphene surfaces are similar to that seen in our experiments. ${ }^{39}$

In Figure 4 we compare the frequencies of the Raman peaks of both the exfoliated and epitaxial graphene samples and their products. Previously, it has been shown that modulating the Fermi level using either an electrostatic or an electrolyte gate can result in a blue shift in the $\mathrm{G}$ band frequency of $1 \mathrm{~L}$ and $2 \mathrm{~L}$ graphene. ${ }^{18,40-42} \mathrm{We}$ observe a similar blue shift in 1-LG but a much larger $\left(22 \mathrm{~cm}^{-1}\right)$ red shift in the product from epitaxial graphene, along with a $60-70 \mathrm{~cm}^{-1}$ red shift of the 2D band. The widths of the spectral peaks do not broaden as is characteristic of disordered graphitic materials. ${ }^{43}$ Taking $1347 \mathrm{~cm}^{-1}$ as the mean D-band frequency of a structural defect in pristine graphene and graphitic materials with long-range crystalline order and $1620 \mathrm{~cm}^{-1}$ as that for the $\mathrm{D}^{*}$ band, the product of the nitrophenyl radical functionalization on thin epitaxial graphene indicates that the introduction of the nitrophenyl groups leads to saturated sites in the graphene lattice that may be viewed as internal edges to the conjugated regions.

Thus, the covalent chemical reorganization of the $\pi$-bonds of graphene directed by the formation of additional C-C bonds at basal plane $\mathrm{sp}^{3}$ centers is a facile route for bandgap engineering of graphene and patterning of electronic circuits on graphene wafers, which is capable of modifying the electronic structure of graphene without the attendant structural defects or the introduction of dangling bonds and functionalitiesthataccompanyphysicalpatterningtechniques.

Acknowledgment. We acknowledge financial support from DOD/DMEA under contract H94003-09-2-0901 and NSF-MRSEC through contract DMR-0820382. ARPES measurements were supported by a Partner University Fund from the Embassy of France. We also thank A. Tejeda, A. Taleb-Ibrahimi, P. Le Fèvre and F. Bertran at the Cassiopée beamline for their expertise in acquiring the ARPES data. Finally, we acknowledge the SOLEIL synchrotron radiation facilities.

Supporting Information Available. Details of sample preparation, Raman microscopy, plot of $\mathbf{I}_{\mathrm{D}} / \mathbf{I}_{\mathrm{G}}$ vs $\mathbf{L}_{\mathrm{D}}$ and $\mathbf{I}_{\mathrm{D}} / \mathbf{I}_{\mathrm{G}}$ vs F( $\left.\mathrm{sp}^{3}\right)$ in a-C:H samples. This material is available free of charge via the Internet at http://pubs.acs.org. 
(1) Berger, C.; Song, Z.; Li, T.; Li, X.; Ogbazghi, A. Y.; Feng, R.; Dai,Z.; Marchenkov, A. N.; Conrad, E. H.; First, P. N.; de Heer, W. A.J. Phys. Chem. B 2004, 108 (52), 19912-19916.

(2) Novoselov, K. S.; Geim, A. K.; Morozov, S. V.; Jiang, D.; Zhang, Y.; Dubonos, S. V.; Grigorieva, I. V.; Firsov, A. A. Science 2004, 306, 666-669.

(3) Lin, Y. M.; Dimitrakopoulos, C.; Jenkins, K. A.; Farmer, D. B.; Chiu, H. Y.; Grill, A.; Avouris, P.

Science 2010, 327 (5966), 662-662.

(4) Nakada, K.; Fujita, M.; Dresselhaus, G.; Dresselhaus, M. S. Phys Rev. B 1996, 54, 17954-17961.

(5) Wakabayashi, K.; Fujita, M.; Ajiki, H.; Sigrist, M. Phys Rev. B 1999,

59, 8271-8282.

(6) Han, M. Y.; Ozyilmaz, B.; Zhang, Y. B.; Kim, P. Phys. Rev. Lett. 2007, 98 (20), 206805-4.

(7) Ryu, S.; Han, M. Y.; Maultzsch, J.; Heinz, T. F.; Kim, P.; Steigerwald, M.; Brus, L. E. Nano Lett. 2008, 8, 4597-4602.

(8) Jiao, L.; Zhang, L.; Wang, X.; Diankov, G.; Dai, H. Nature 2009,

458, 877-880.

(9) Jiao, L.; Wang, X.; Diankov, G.; Wang, H.; Dai, H. Nat. Nanotechnol. 2010, 5, 321-325.

(10) Kosynkin, D. V.; Higginbotham, A. L.; Sinitskii, A.; Lomeda, J. R.; DImiev, A.; Price, B. K.; Tour, J. M. Nature 2009, 458, 872-876.

(11) Bekyarova, E.; Itkis, M. E.; Ramesh, P.; Berger, C.; Sprinkle, M.;

de Heer, W. A.; Haddon, R. C. J. Am. Chem. Soc. 2009, 131, 1336- 1337.

(12) Bekyarova, E.; Itkis, M. E.; Ramesh, P.; Haddon, R. C. Phys. Status Solidi RRL 2009, 3, $184-186$.

(13) Hu, H.; Zhao, B.; Hamon, M. A.; Kamaras, K.; Itkis, M. E.; Haddon, R. C. J. Am. Chem. Soc. 2003,

125, 14893-14900.

(14) Kamaras, K.; Itkis, M. E.; Hu, H.; Zhao, B.; Haddon, R. C. Science 2003, 301, 1501.

(15) Sprinkle, M.; Siegel, D.; Hu, Y.; Hicks, J.; Tejeda, A.; Taleb-Ibrahimi, A.; LeFevre, P.; Bertran, F.;

Vizzini, S.; Enriquez, H.;Chiang, S.; Soukiassian, P.; Berger, C.; de Heer, W. A.; Lanzara,A.; Conrad, E. H.

Phys. Rev. Lett. 2009, 103, 226803-1-226803-4.

(16) Coletti, C.; Riedl, C.; Lee, D. S.; Krauss, B.; Patthey, L.; vonKlitzing, K.; Smet, J. H.; Starke, U. Phys. Rev. B 2010, 81, 235401-1-235401-8.

(17) Ferrari, A. C.; Meyer, J. C.; Scardaci, V.; Casiraghi, C.; Lazzeri, M.;Mauri, F.; Piscanec, S.; Jiang, D.; Novoselov, K. S.; Roth, S.; Geim

(18) Malard, L. M.; Pimenta, M. A.; Dresselhaus, G.; Dresselhaus, M. S.Phys. Rep. 2009, 473, 51-87.

(19) Mapelli, C.; Castiglioni, C.; Zerbi, G.; Mullen, K. Phys. Rev. B 1999,60 (18), 12710-12725.

(20) Sharma, R.; Baik, J. H.; Perera, C. J.; Strano, M. S. Nano Lett. 2010,10, 398-405.

(21) Koehler, F. M.; Jacobsen, A.; Ensslin, K.; Stampfer, C.; Stark, W. J.Small 2010, 6 (10), 1125-1130.

(22) Farmer, D. B.; Golizadeh-Mojarad, R.; Perebeinos, V.; Lin, Y.-M.;Tulevski, G. S.; Tsang, J. C.;

Avouris, P. Nano Lett. 2009, 9 (1),388-392.

(23) Liu, Y. C.; Mccreery, R. L. J. Am. Chem. Soc. 1995, 117 (45), 11254-11259.

(24) Hass, J.; de Heer, W. A.; Conrad, E. H. J. Phys.: Condens. Matter2008, 20, 323202.

(25) Faugeras, C.; Nerriere, A.; Potemski, M.;Mahmood, A.; Dujardin,E.; Berger, C.; de Heer, W. A. Appl. Phys. Lett. 2008, 92 (1), No.011914-3.

(26) Riedl, C.; Starke, U.; Bernhardt, J.; Franke, M.; Heinz, K. Phys.Rev. B 2007, 76, 245406-1-245406-8.

(27) Hass, J.; Varchon, F.; Millan-Otoya, J. E.; Sprinkle, M.; Sharma,N.; de Heer, W. A.; Berger, C.; First,

P. N.; Magaud, L.; Conrad,E. H. Phys. Rev. Lett. 2008, 100 (12), 125504-4.

(28) Poncharal, P.; Ayari, A.; Michel, T.; Sauvajol, J.-L. Phys. Rev. B2008, 78, 113407.

(29) Boukhvalov, D. W.; Katsnelson, M. I. Phys. Rev. B 2008, 78, No.085413.

(30) Balog, R.; Jorgensen, B.; Nilsson, L.; Andersen, M.; Rienks, E.;Bianchi, M.; Fanetti, M.; Laegsgaard, E.; Baraldi, A.; Lizzit, S.;Slijivancanin, Z.; Besenbacher, F.; Hammer, B.; Pedersen, T. G.;Hofmann, P.; Hornekaer, L. Nat. Mater. 2010, 9, 315-319.

(31) Delgado, J. C.; Herrera, J. M. R.; Jia, X.; Cullen, D. A.; Muramatsu,H.; Kim, Y. A.; Hayashi, T.; Ren, Z.; Smith, D. J.; Okuno, Y.; Ohba,T.; Kanoh, H.; Kaneko, K.; Endo, M.; Terrones, H.; Dresselhaus,M. S.;

Terrones, M. Nano Lett. 2008, 8 (9), 2773-2778.

(32) Elias, D. C.; Nair, R. R.; Mohiuddin, T. M. G.; Morozov, S. V. B.,P.; Halsall, M. P.; Ferrari, A. C.;

Boukhvalov, D. W.; Katsnelson,M. I.; Geim, A. K.; Novoselov, K. S. Science 2009, 323, 610-613.

(33) Luo, Z.; Yu, T.; Kim, K.-j.; Ni, Z.; You, Y.; Lim, S.; Shen, Z.; Wang,S.; Lin, J. ACS Nano 2009, 3 (7), 1781-1788.

(34) Thomsen, C.; Reich, S. Phys. Rev. Lett. 2000, 85 (24), 5214-5217. 
(35) Tuinstra, F.; Koenig, J. L. J. Chem. Phys. 1970, 53 (3), 1126-1130.

(36) Ferrari, A. C.; Robertson, J. Phys. Rev. B 2000, 61 (20), 14095-14107.

(37) Lucchese, M. M.; Stavale, F.; Ferreira, E. H. M.; Vilani, C.;

Moutinho, M. V. O.; Capaz, R. B.; Achete, C. A.; Jorio, A. Carbon2010, 48, 1592-1597.

(38) Jiang, D.; Sumpter, B. G.; Dai, S. J. Phys. Chem. B 2006, 110,23628-23632.

(39) Kudin, K. N.; Ozbas, B.; Schniepp, H. C.; Prud'homme, R. K.;Aksay, I. A.; Car, R. Nano Lett. 2008, 8

(1), 36-41.

(40) Pisana, S.; Lazzeri, M.; Casiraghi, C.; Novoselov, K. S.; Geim, A. K.;Ferrari, A. C.; Mauri, F. Nat. Mater. 2007, 6, 198-201.

(41) Das, A.; Pisana, S.; Chakraborty, B.; Piscanec, S.; Saha, S. K.;Waghmare, U. V.; Novoselov, K. S.; Krishnamurthy, H. R.; Geim,A. K.; Ferrari, A. C.; Sood, A. K. Nature Nanotechnol. 2008, 3 (4),210-215.

(42) Yan, J.; Henriksen, E. A.; Kim, P.; Pinczuk, A. Phys. Rev. Lett.2008, 101, 136804-1136804-4.

(43) Nemanich, R. J.; Solin, S. A. Phys. Rev. B 1979, 20 (2), 392-401. 


\section{Figure Captions}

FIGURE 1. Characteristics of nitrophenyl (NP) functionalized exfoliated graphene. (a) Schematic of the reaction of p-nitrobenediazonium tetrafluoroborate with graphene showing the initial nitrophenyl (NP) functionalized graphene product. (b) Raman spectrum (with $\lambda \mathrm{EX}$ ) $532 \mathrm{~nm}$ ) of pristine exfoliated graphene and NP-functionalized exfoliated graphene. The inset shows an optical image of the graphene sample attached to a thicker graphene flake. (c) Raman spectrum of a bilayer exfoliated graphene sample and the same sample after the NP-functionalization reaction. The inset shows fitting of the $2 \mathrm{D}$ peak to four Lorentzians. (d) Spectra of the NP-functionalized graphene and bilayer graphene samples showing peaks with identical frequencies. ID/IG $)^{2.6}$ for the NP-functionalized graphene spectrum shown and the fwhm of the $\mathrm{G}$ and $\mathrm{D}$ bands are 23 and $28 \mathrm{~cm}-1$, respectively. (e) AFM height images of pristine and NPfunctionalized graphene showing the increased surface roughness after functionalization.

FIGURE 2. Characteristics of nitrophenyl (NP) functionalized epitaxial graphene. (a) Raman spectrum of NP-functionalized epitaxial graphene; the inset is an optical image of epitaxial graphene on the C-face $\mathrm{SiC}$. $\mathrm{ID}_{\mathrm{D}} \mathbf{I}_{\mathrm{G}}=2.6$ and the fwhm of the $\mathrm{G}$ and $\mathrm{D}$ bands are 35 and $33 \mathrm{~cm}^{-1}$, respectively. (b,c) Raman spectra of pristine epitaxial graphene samples. The fwhm of the $\mathrm{G}$ bands in these two spectra are 15 and $14 \mathrm{~cm}^{-1}$ respectively. The spatial distribution of the Raman profile varies between these two extremes across the wafer; the inset in panel b shows the 2D peak splitting pattern when fitted to four Lorentzians; in panel c, the single component 2D band has a fwhm of $18 \mathrm{~cm}^{-1}$. (d,e) AFM height images of epitaxial graphene before and after NP-functionalization, respectively, showing increased surface roughness after functionalization.

FIGURE 3. The band gap in nitrophenyl-functionalized epitaxial graphene measured using ARPES. (a) ARPES measured NP-functionalized graphene band structure perpendicular to the $\Gamma$-K direction at the graphene K-point (incident photon energy is $36 \mathrm{eV}$ ). Two diffuse bands representing gapped Dirac cones are visible; dashed lines are used to highlight the bands. From the $\Delta \mathrm{k}$ broadening of the bands we estimate that the coherent domain size of the functionalized epitaxial graphene is $\sim 2 \mathrm{~nm}$. (b) Constant energy cuts from panel a for different values of ky (curves are vertically displaced). Dashed curve show the cones with the band edge $0.36 \mathrm{eV}$ below EF.

FIGURE 4. Changes in the Raman spectra in exfoliated and epitaxial graphene due to NP-functionalization. The individual spectra have been offset vertically for clarity. The observation of the sharp Alg mode peak along with the shifted $\mathrm{G}$ and 2D bands is characteristic of a reorganized periodicity of the $\pi$-bonds in nitrophenyl functionalized graphene. In the NP-functionalized epitaxial graphene spectrum, $\mathbf{I} \mathrm{D} / \mathbf{I G}_{\mathrm{G}}=3.3$. The inset shows the theoretical curve derived from a numerical fit to selected data in the literature, (see Supporting Information Figure S1) relating the variation of $\mathbf{I}_{\mathrm{D}} / \mathbf{I}_{\mathrm{G}}$ to the fractional sp3 content $\left[\mathrm{F}\left(\mathrm{sp}^{3}\right)\right]$ in a$\mathrm{C}: \mathrm{H}^{\cdot 36}$ 
a

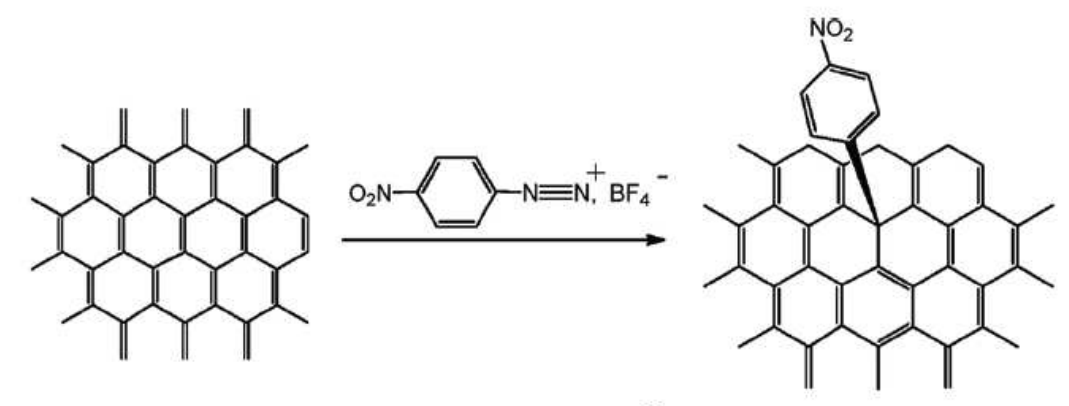

b Exfoliated graphene
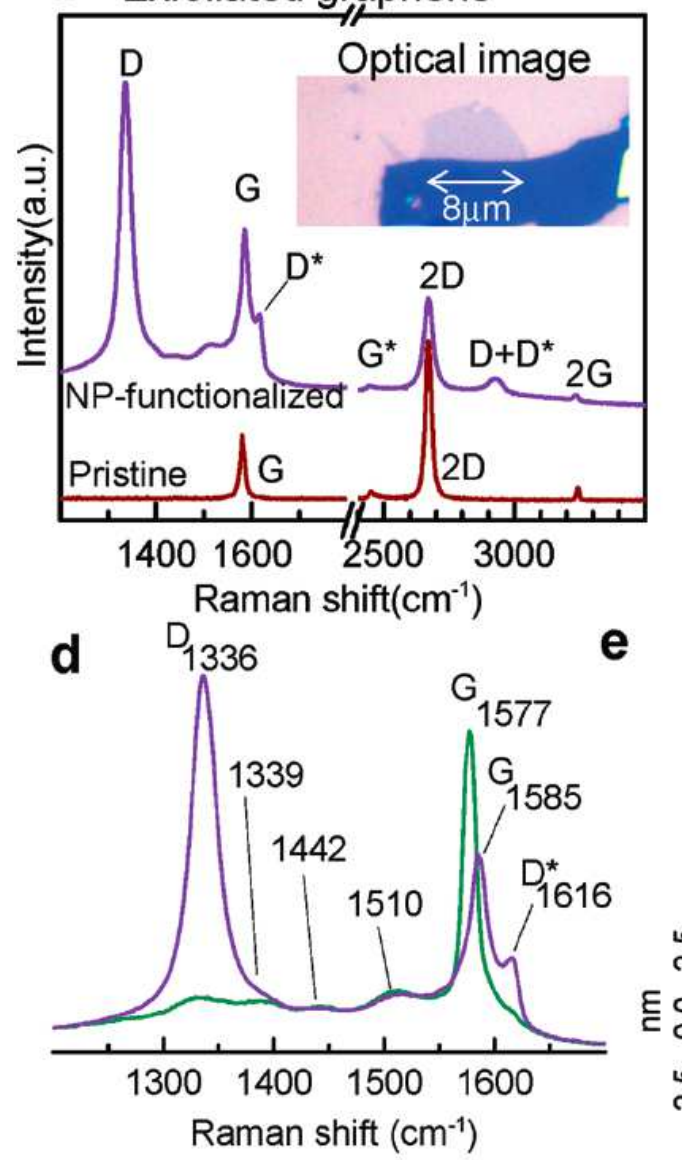

C Exfoliated bilayer graphene
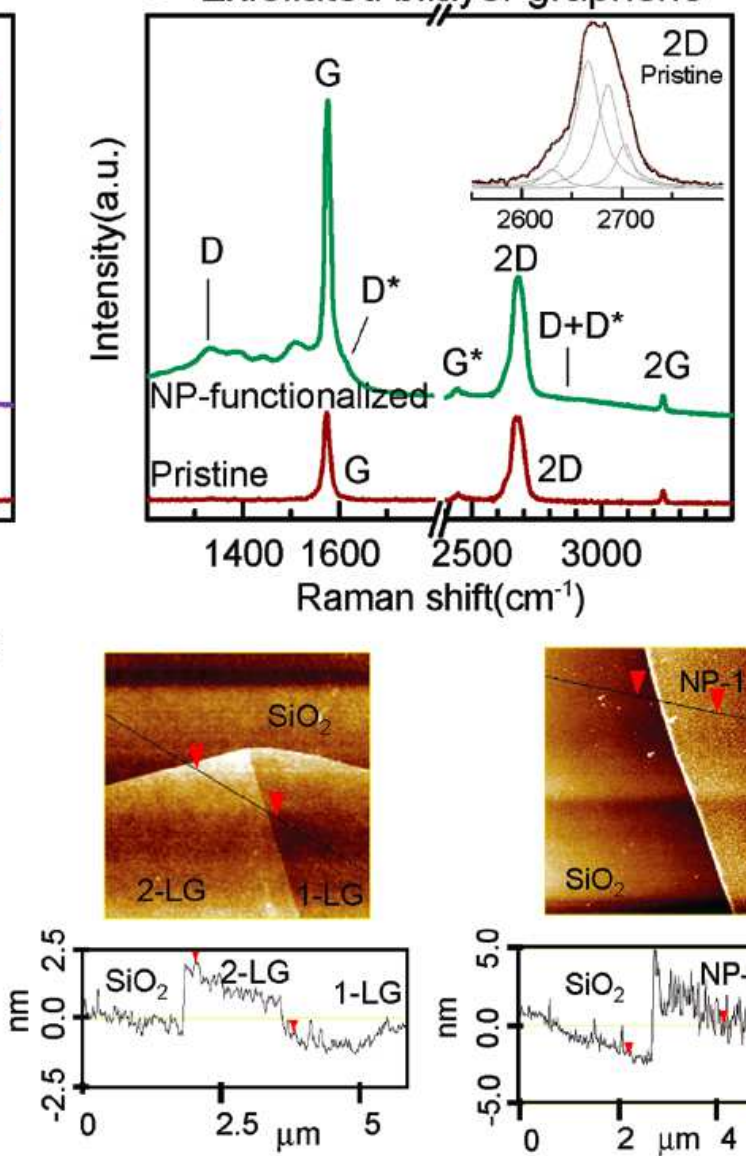

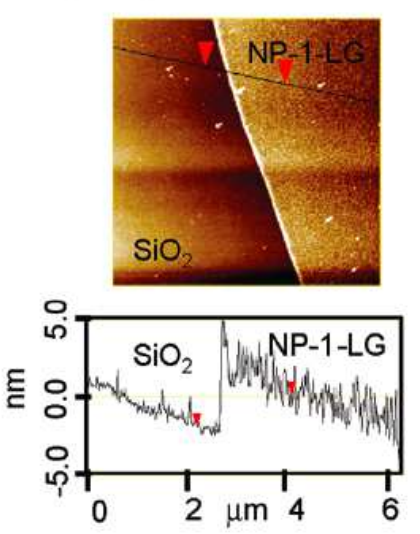

Figure 1 


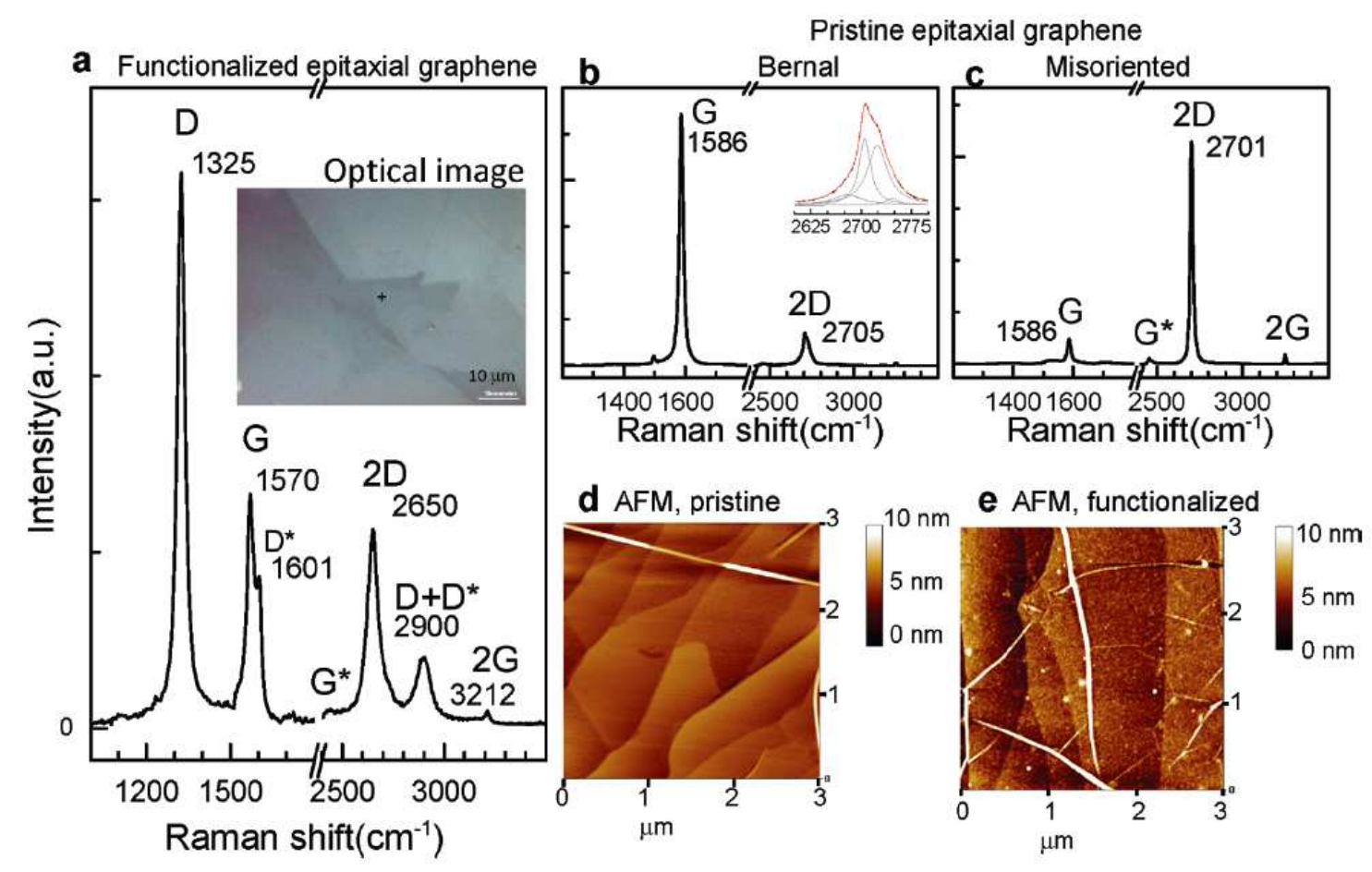

Figure 2
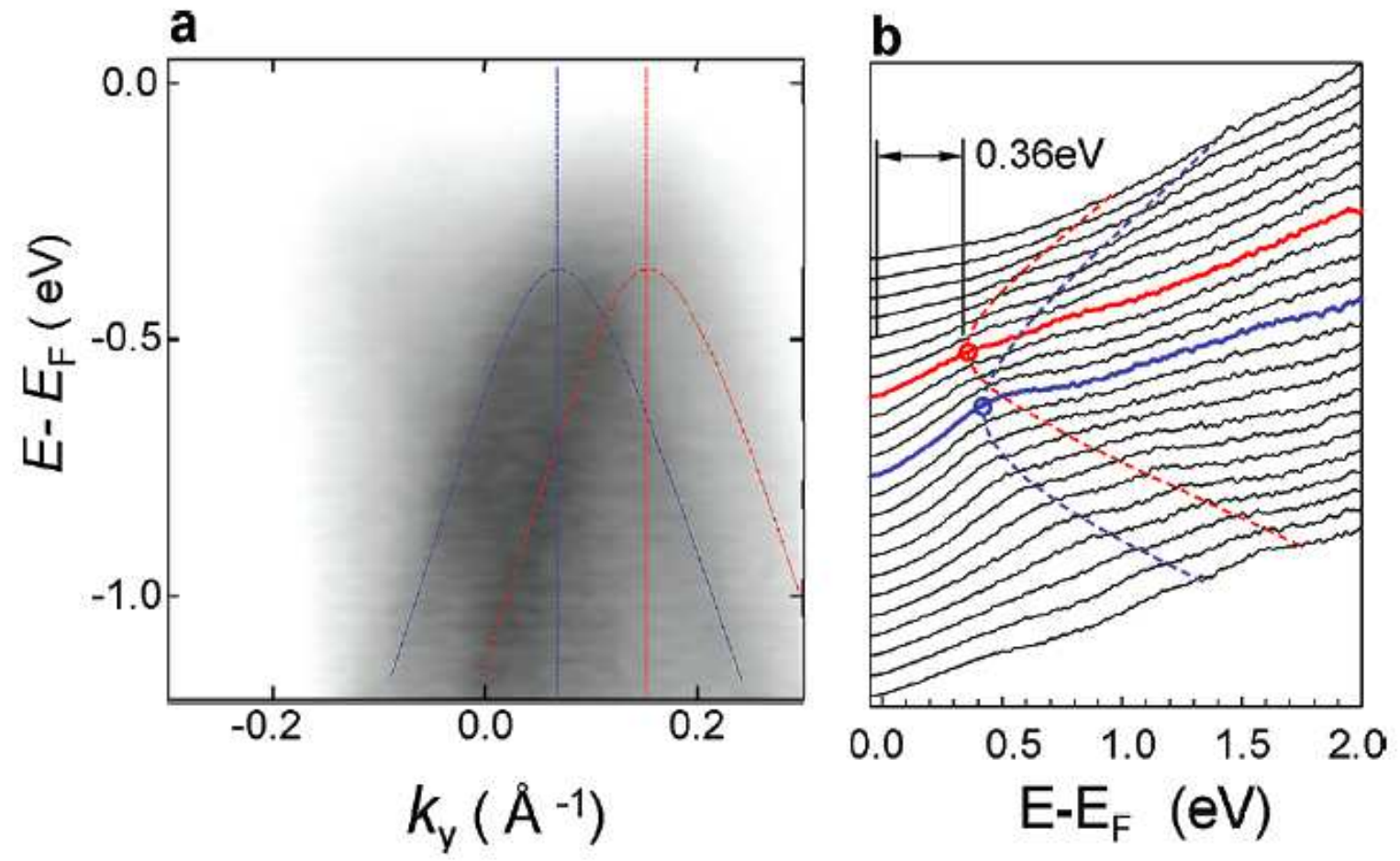

Figure 3 


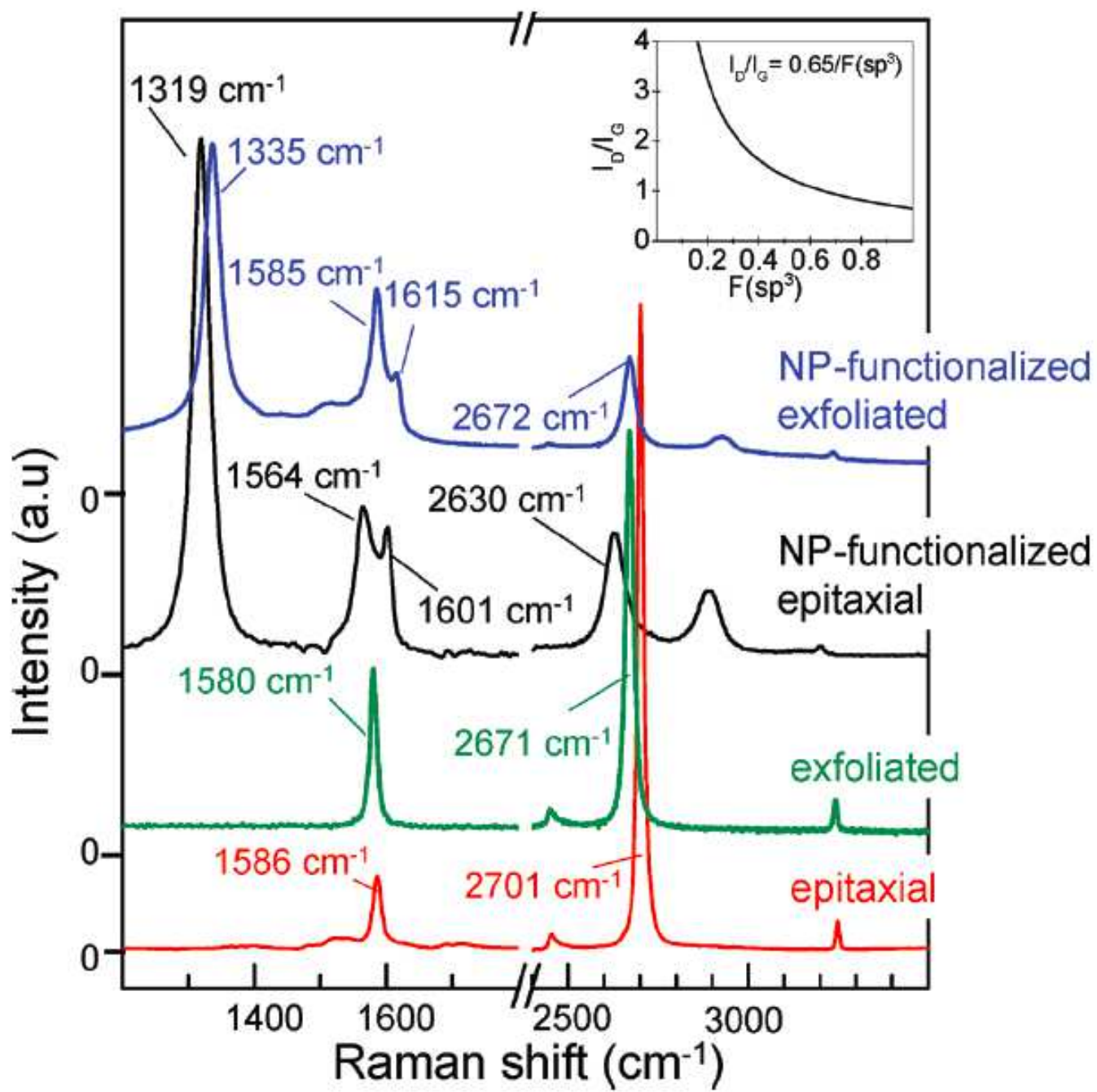

Figure 4 